\title{
Unification of the physics of nucleons and nuclei
}

\author{
Ulf-G. Meißner ${ }^{\mathrm{a}}$
}

${ }^{a}$ Universität Bonn, HISKP (Th), Nußallee 12-14, D-53115 Bonn, Germany

I outline an ambitious program which aims to achieve a unified description of nucleon and nuclear properties based on one chiral effective field theory.

\section{WHAT AND WHY?}

Tremendous progress has been made in calculating the properties of few-nucleon systems to a very high level of accuracy, as witnessed by many talks during this conference. In particular, precise calculations of few-body systems are needed since light nuclei are (almost) the only laboratory for extracting the elusive properties of the neutron. However, the highly successful so-called standard approach has reached certain limitations, for example two- and three-nucleon forces are not calculated consistently with each other and the physics underlying the nucleon structure is treated very differently from the one leading to the nuclear bound and scattering states. This induces some theoretical uncertainty, which is difficult to quantify. For these and other reasons, we really would like to have a description of nucleons and nuclei from one field theory. Such a scheme must be rooted in the symmetries of QCD and, being based on field theory, should allow for a straightforward implementation of gauge and other symmetries. Furthermore, we wish to have at our disposal some small parameter(s), leading to a systematic and controlled expansion. Consequently, theoretical error bars take over the often (mis)used $\chi^{2} /$ dof $=$ 1 criterion. Furthermore, such a scheme should be extendable to cope with inelastic processes and the onset of relativity. This can be formulated as a new paradigm: To calculate nucleon as well as nuclear properties and dynamics, use quantum field theory and organize the theory in a systematic expansion based on (a) small parameter(s). Needless to say that the working tool will be an appropriately tailored effective field theory (EFT). Now one might ask the question whether such a program is not too ambitious from the beginning? I do not believe so, chiefly because QCD itself offers a rich and intricate pattern of symmetries and their violations to achieve this goal. More precisely, the spontaneous, explicit and anomalous (chiral) symmetry breaking that QCD is supposed to undergo can be analyzed in terms of EFT. Furthermore, the recent developments in formulating a nuclear EFT allow for the required link between nucleon and nuclear physics, as I will show in the following sections. In this short write-up, I can only give a flavor of these developments but I hope that the reader will become sufficiently curious. For more introductory material and reviews on these various intertwined topics I refer to the "handbook of QCD" [1]. 


\section{HOW ?}

For the light quark flavors, the QCD Hamiltonian possesses an approximate chiral symmetry. In the chiral limit of vanishing quark masses, this symmetry is exact. It is, however, not realized in the ground state or the particle spectrum. Rather, the chiral symmetry is broken down to its vectorial subgroup with the appearance of three pseudoscalar Goldstone bosons, the pions (I restrict myself here to the two flavor case). If one considers reactions with at most one nucleon in the asymptotic in- and the out-states, a perturbative expansion in terms of small momenta and quark masses is possible. Denoting by $Q$ a generic small parameter, any $\mathrm{S}$-matrix element or transition current can be written as

$\mathcal{M}=\sum_{\nu} \mathcal{M}_{\nu}, \quad$ with $\quad \mathcal{M}_{\nu}=Q^{\nu} f\left(Q / \lambda, g_{i}\right)$

where $\lambda$ is a renormalization scale, $g_{i}$ denotes coupling (low-energy) constants of the contact interactions appearing at each order, and the function $f$ is of order one. The important observation is that the index $\nu$ is bounded from below because of chiral symmetry. Thus, provided $Q$ is parametrically small, this defines a perturbative expansion in terms of tree and (pion) loop graphs (see also the discussion on the structure of the nucleon below). For systems with baryon number larger than one, an additional non-perturbative resummation is necessary to generate large scattering lengths and shallow nuclear bound states, as described in the talks by Beane [2] and Epelbaum [3] at this conference. Next, I briefly discuss the structure of the effective chiral Lagrangian, which decomposes into the purely mesonic, the meson-nucleon and the nucleonic sectors. It has the generic form

$\mathcal{L}_{\text {eff }}=\mathcal{L}_{\pi \pi}+\mathcal{L}_{\pi N}+\mathcal{L}_{N N}+\ldots$,

where the ellipsis stands for terms with six or more nucleons fields. Each of these terms has a chiral expansion, for example $\mathcal{L}_{\pi N}=\mathcal{L}_{\pi N}^{(1)}+\mathcal{L}_{\pi N}^{(2)}+\mathcal{L}_{\pi N}^{(3)}+\ldots$, where the superscript denotes the chiral dimension. At each order, new and more contact interactions accompanied by so-called low-energy constants (LECs) appear. While the number of such terms quickly rises as one goes to higher orders, the number of operators contributing to a certain process stays manageable. Consider e.g. pion-nucleon scattering, analyzed in terms of the chiral effective pion-nucleon Lagrangian. We have to determine 4, 5, and 4 LECs at dimension two, three and four, respectively, from the total of 7, 23 and 118 terms at these orders [4]. Furthermore, the numerical values for these LECs are understood in many cases, rooted in the so-called resonance saturation hypothesis (that is integrating out heavier degrees of freedom and estimating LECs from the couplings and masses of these heavy states), first investigated in the meson sector $[5,6]$. As an example, take the finite dimension two coefficient $c_{4}$ from $\mathcal{L}_{\pi N}^{(2)}$. From the analysis of various $\pi N$ scattering PWAs, one finds $c_{4}=3.36 \ldots 3.73 \mathrm{GeV}^{-1}$. In terms of resonance saturation [7] (see Fig. 1), we get $c_{4}=c_{4}^{\Delta}+c_{4}^{R}+c_{4}^{\rho}=(1.9+0.1+1.6) \mathrm{GeV}^{-1}=3.6 \mathrm{GeV}^{-1}$, where $\Delta, R$ and $\rho$ denote the Delta, the Roper resonance and the $\rho$ meson, in order. We thus see that not only the numerical value of this LEC can be understood but also it comes out somewhat larger than the value based on naive dimensional analysis (NDA), $\left|c_{4}^{\mathrm{NDA}}\right| \simeq 1 / m \simeq 1 \mathrm{GeV}^{-1}$ (with $m$ the nucleon mass). For a similar investigation of the LECs in $\mathcal{L}_{N N}$ (four-nucleon operators) see [8]. 


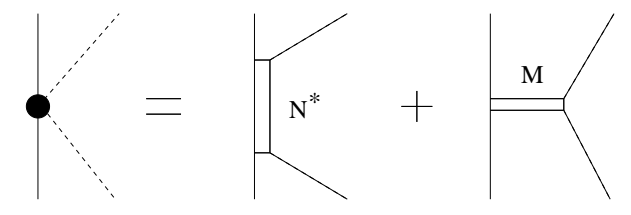

Figure 1. Resonance saturation for a dimension two operator (filled circle). Solid and dashed lines stand for nucleons and pions, $N^{*}(M)$ denotes a baryon (meson) resonance.

\section{FIRST EXAMPLE: NUCLEON AND DEUTERON ELECTROMAGNETIC FORM FACTORS}

As a first example how such a unified approach works, we consider elastic electron scattering off the nucleon and off deuterium. First, we discuss the nucleon case. The nucleon matrix element of the conserved vector current is parameterized in terms of two form factors $F_{1,2}$ (or, equivalently, the Sachs form factors, $G_{E, M}$ ). These form factors are also a good example of how the complicated structure of the nucleon, which is certainly an extended object, arises from the structureless spin- $1 / 2$ fields in the chiral Lagrangian. There are two distinct contributions. The first finite size effect is related to the already mentioned fact that the effective Lagrangian contains all terms allowed by PCT transformations and chiral symmetry. This leads to a string of terms of higher order couplings in the various sectors. These couplings parameterize all short distance physics contributing to the internal structure of the baryons.

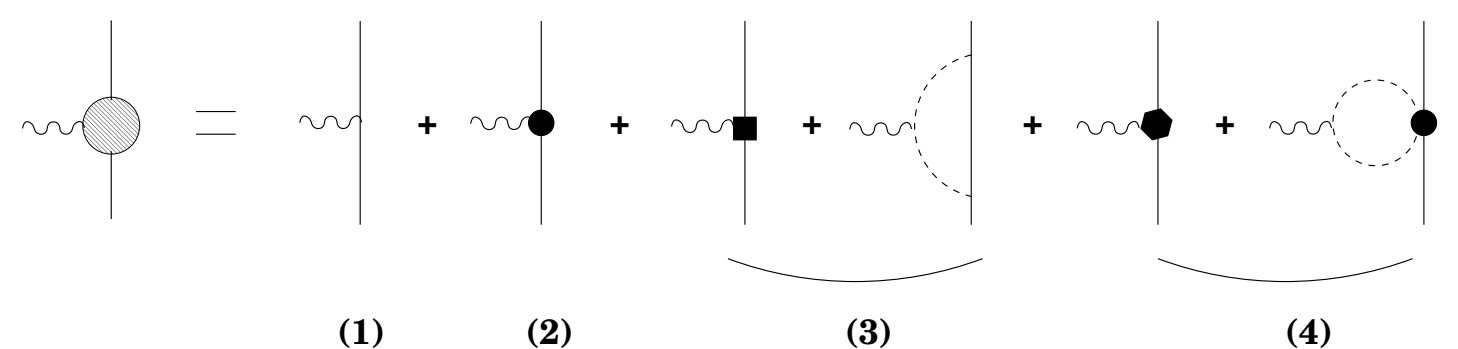

Figure 2. Chiral expansion of a nucleon electromagnetic form factor. The lowest order graph (1) with dimension one insertions only gives the charge of the baryon. Tree graphs with insertions from the dimension two (2), three (3) and four (4) effective Lagrangian are depicted by the solid circle, square and sextangle, in order. Pion loop graphs start at third order (3), and fourth order loop graphs (4) have exactly one dimension two insertion. Only one typical loop graph at the orders considered is shown. Solid, dashed and wiggly lines denote nucleons, pions and photons, respectively.

The other finite size effect of the nucleon only starts to get generated at the one-loop level, as in every quantum field theory short-lived fluctuations can occur. In baryon CHPT a nucleon typically emits a pion, this energetically forbidden $\pi \mathrm{N}$ intermediate state lives for a short while and then the pion is reabsorbed by the nucleon, in accordance with the uncertainty principle. This mechanism is responsible for the venerable old idea of 

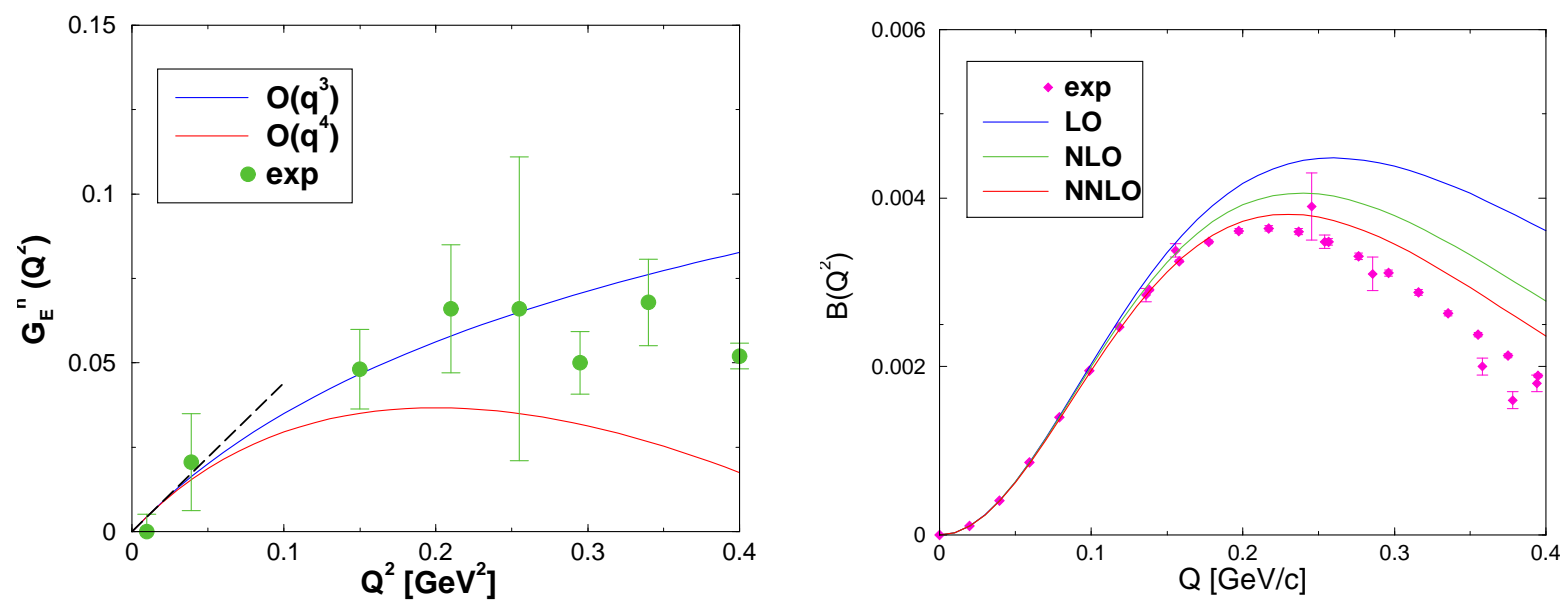

Figure 3. Left panel: Neutron electric form factor $G_{E}^{n}\left(Q^{2}\right)$ at third and fourth order in comparison to the data, see [10]. Right panel: Deuteron structure function $B$ versus the photon virtuality $Q$ at LO, NLO and NNLO in comparison to the data, see [12].

the "pion cloud" of the nucleon, which in CHPT can be put on the firm ground of field theoretical principles. This is graphically depicted and further explained in Fig. 2. It is, however, important to stress that the relative strength of these contributions in general depends on the choice of renormalization scale, for a detailed discussion I refer to [9]. In the left panel of Fig. 3 I show the result for the neutron electric form factor based on a fourth order calculation employing infrared regularization [10] in comparison to modern data extracted from polarization experiments at NIKHEF, MAMI and MIT-Bates. There is one LEC, which can be fixed from the neutron charge radius measured in thermal neutron-atom scattering as indicated by the dashed line in the figure. As discussed in detail in [10], to achieve an equally accurate description of the other three form factors for $Q^{2} \lesssim 0.4 \mathrm{GeV}^{2}$, vector mesons must be included. For these 3-point functions, that can be done systematically and with no new tuneable parameters. Space forbids to show the resulting form factors, the interested reader should consult Ref. [10]. Let us now turn to electron scattering off the deuteron. The central object is the (unpolarized) scattering cross section, which is given in terms of two structure functions,

$\frac{d \sigma}{d \Omega}=\left(\frac{d \sigma}{d \Omega}\right)_{\text {Mott }}\left[A\left(q^{2}\right)+B\left(q^{2}\right) \tan \frac{\theta}{2}\right]$,

with $q^{2}=-Q^{2}<0$ the invariant momentum transfer squared and $\theta$ is the scattering angle in the centre-of-mass frame. Furthermore, we have separated the QED (Mott) cross section. These structure functions are subject to a chiral expansion, and consist of essentially two types of contributions. The first type of terms comprise the so-called impulse approximation contribution, which is nothing but the embedding of the single nucleon form factors within the deuteron, as indicated by the dashed boxes in the upper row of Fig. 4. In addition, there are the so-called three-body corrections (meson exchange currents), which are parameter-free up to NLO and have one four-nucleon-photon operator 
at NNLO $[11,12]$. The corresponding LEC can be fixed at zero momentum transfer from the deuteron magnetic moment. In the right panel of Fig. 3 the structure function $B(Q)$ is shown at LO, NLO and NNLO. Visibly, the description of the data improves at higher orders and, furthermore, the corrections become smaller when going from LO to NLO to NNLO. As stressed in [12], the limiting factor in the accuracy to be achieved for the deuteron structure functions stems from the description of the single nucleon form factors. For a somewhat different approach, see [13].

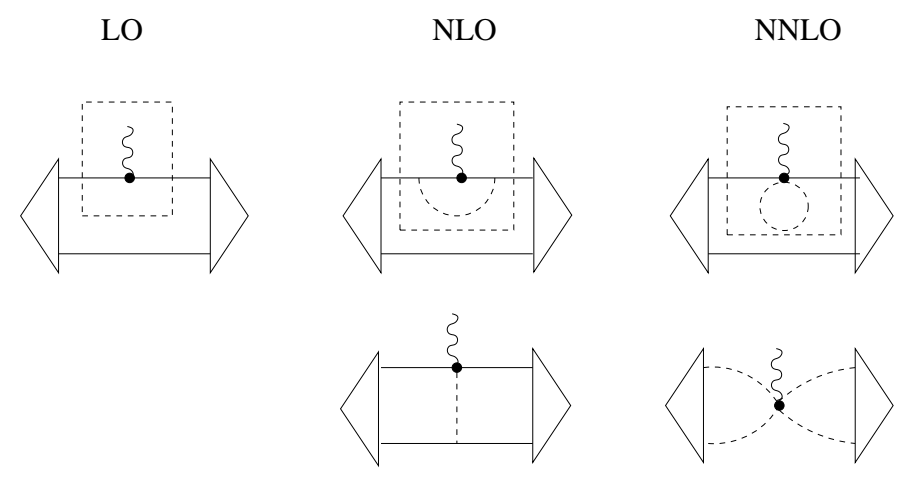

Figure 4. Chiral expansion of ed scattering. The upper row refers to the single scattering contributions (impulse approximation), the lower row to the three-body corrections (meson exchange currents). The triangle symbolizes the deuteron wave function, for further notation see Fig. 2. Only one representative diagram is shown at each order.

While not very spectacular, I think that this example nicely shows how indeed the nucleon and nuclear properties can arise from one effective field theory and it will be of utmost importance to extend this program to the investigation of the electromagnetic structure of light nuclei.

\section{SECOND EXAMPLE: PION-NUCLEON AND PION-DEUTERON SCATTERING}

Another interesting example concerns pion-nucleon scattering at zero energy, i.e. the S-wave scattering lengths $a^{+}$(isoscalar) and $a^{-}$(isovector). Already Weinberg pointed out many decades ago that these observables allow for a crucial test of chiral symmetry breaking. In the mean time, the chiral expansion of these quantities has been worked out to fourth order $[14,15]$ (for brevity, we do not display the fourth order contribution to $a^{+}$ but refer the reader to [15])

$$
\begin{aligned}
& 4 \pi(1+\mu) a^{+}=\frac{M_{\pi}^{2}}{F_{\pi}^{2}}\left(\Delta-\frac{g_{A}^{2}}{4 m}\right)+\frac{3 g_{A}^{2} M_{\pi}^{3}}{64 \pi F_{\pi}^{4}}+\mathcal{O}\left(M_{\pi}^{4}\right) \\
& 4 \pi(1+\mu) a^{-}=\frac{M_{\pi}}{2 F_{\pi}^{2}}+\frac{4 M_{\pi}^{3}}{F_{\pi}^{2}}\left(\bar{D}+\frac{g_{A}^{2}}{32 m^{2}}\right)+\frac{M_{\pi}^{3}}{16 \pi^{2} F_{\pi}^{4}}+\mathcal{O}\left(M_{\pi}^{5}\right)
\end{aligned}
$$

where $\mu=M_{\pi} / m \simeq 1 / 7$ is the small threshold parameter, $\Delta=-4 c_{1}+2 c_{2}+2 c_{3}$ and $\bar{D}=\bar{d}_{1}+\bar{d}_{2}+\bar{d}_{3}+2 \bar{d}_{5}$ are combinations of dimension two and three LECs, respectively. These can be determined by a fit to the existing PWAs at finite energy, so that one can predict the scattering lengths and the phases at higher energies. This leads to the results collected in Table 1 [15]. We see that for $a^{-}$the convergence is good and the value has a small theoretical uncertainty (the low value from Fit 2 should be excluded as discussed in detail in [16]). On the other hand, the convergence for $a^{+}$is moderate and the theoretical error quite large. These results are consistent with the precise pionic 
Table 1

Convergence of the S-wave scattering lengths for CHPT fits to the Karlsruhe (Fit 1), Zürich (Fit 2), and VPI (Fit 3) phase-shift analyses of $\pi N$ data. $O\left(p^{n}\right)$ means that all terms up-to-and-including order $n$ were included. Units are $M_{\pi}^{-1}$.

\begin{tabular}{|rr|r|r|r|r|}
\hline & & $O(p)$ & $O\left(p^{2}\right)$ & $O\left(p^{3}\right)$ & $O\left(p^{4}\right)$ \\
\hline \hline \multirow{3}{*}{$a^{+}$} & Fit 1 & 0.0 & 0.0046 & -0.0100 & -0.0096 \\
& Fit 2 & 0.0 & 0.0024 & 0.0049 & 0.0045 \\
& Fit 3 & 0.0 & 0.0101 & 0.0014 & 0.0027 \\
\hline \multirow{3}{*}{$a^{-}$} & Fit 1 & 0.0790 & 0.0790 & 0.0905 & 0.0903 \\
& Fit 2 & 0.0790 & 0.0790 & 0.0772 & 0.0771 \\
& Fit 3 & 0.0790 & 0.0790 & 0.0870 & 0.0867 \\
\hline
\end{tabular}

hydrogen data obtained at PSI, which again do not allow to pin down $a^{+}$very precisely (see the discussion in [17]). It is also important to stress that only with the recent work of the Bern group [18] we really have a clear-cut analysis of the isospin breaking effects that are so important to properly deduce the strong contribution to the energy shift of the $\pi^{-} p$ atom (note also that the conventional methods of subtracting the electromagnetic effects from low energy $\pi N$ scattering data has been put in doubt recently [19]). On the other hand, the deuteron is an isoscalar target and thus to leading order entirely sensitive to $a^{+}$. Beyond leading order, rescattering and other type of three-body corrections play a role, and these are also sensitive to the isovector scattering length. This problem has already been addressed to third order in Refs. [20,21] and to fourth order in [17]. Surprisingly, at fourth order there is only one novel type of correction related to boosting the single scattering amplitudes (cf. the contribution $a_{\text {boost }}$ in Fig. 5), all three-body corrections at this order vanish (for details see [17]). However, as it is known since long, the rescattering contribution (the graph $a_{3-\text { body }}^{2 a}$ in Fig. 5) is much larger than the other terms at this order (the graphs $a_{3-\text { body }}^{2 b c}$ in Fig. 5), see e.g. [22]. On the other hand, in the standard CHPT counting, numerically large contributions like e.g. multiple rescattering (the graph $a_{3 \text {-body }}^{5}$ in Fig. 5) are suppressed. Consequently, one has to deal in a systematic fashion with this issue. This was done in [23], were it was shown that the rescattering type graphs are IR enhanced due to the small binding momentum in the deuteron,

$\gamma=\sqrt{m_{D} E_{D} / 2}=45 \mathrm{MeV} \ll M_{\pi} \simeq 140 \mathrm{MeV}$

with $m_{D}\left(E_{D}\right)$ the deuteron mass (binding energy). More precisely, whenever the exchanged pions can go on (close to) their mass shell, as it is the case for zero energy scattering, one has an additional scale to cope with. This can be accounted for by a modified power counting, where one counts typical momenta in the deuteron as order $p^{2}$, with $p$ the generic small parameter of CHPT. Amplitudes with powers of $M_{\pi}$ are then suppressed and, denoting by $Q$ the small parameter in the modified counting, one obtains an ordering of the various contributions that is consistent with the numerical evaluation of the different mechanisms, as depicted in Fig. 5. Within this new scheme, there are only a few more diagrams to calculate. At $\mathcal{O}\left(Q^{3}\right)$, one has graphs like $2 a$ with either two dimension two or one dimension three (contact term and loop) insertion. At $\mathcal{O}\left(Q^{4}\right)$, the 


\begin{tabular}{|c|c|c|c|c|}
\hline CONTR. & GRAPH & $\begin{array}{l}\text { STANDARD } \\
\text { COUNTING }\end{array}$ & $\begin{array}{l}\text { MODIFIED } \\
\text { COUNTING }\end{array}$ & VALUE \\
\hline$a_{3-\text { body }}^{2 a}$ & & $O\left(p^{3}\right)$ & $O\left(Q^{1}\right)$ & $-1.9 \cdot 10^{-2}$ \\
\hline$a_{\text {boost }}$ & & $O\left(p^{4}\right)$ & $O\left(Q^{3}\right)$ & $4.5 \cdot 10^{-3}$ \\
\hline$a_{3-\text { body }}^{5}$ & & $O\left(p^{5}\right)$ & $O\left(Q^{4}\right)$ & $1.8 \cdot 10^{-3}$ \\
\hline$a_{3-b o d y}^{2 b c}$ & & $O\left(p^{3}\right)$ & $O\left(Q^{5}\right)$ & $-2 \cdot 10^{-4}$ \\
\hline
\end{tabular}

Figure 5. Various contributions to the real part of the $\pi d$ scattering length as explained in the text. The filled circle denotes an insertion from $\mathcal{L}_{\pi N}^{(2)}$.

only new contribution stems from $a_{3-\text { body }}^{5}$, cf. Fig. 5 . With that, the final formula for the real part of $\pi \mathrm{d}$ scattering length, valid to $O\left(Q^{4}\right)$ in the modified power counting, is

$$
\begin{aligned}
\operatorname{Re} a_{\pi d}= & 2 \frac{(1+\mu)}{(1+\mu / 2)}\left(a^{+}+(1+\mu)\left[\left(a^{+}\right)^{2}-2\left(a^{-}\right)^{2}\right] \frac{1}{2 \pi^{2}}\left\langle\frac{1}{\vec{q}^{2}}\right\rangle_{\mathrm{wf}}\right. \\
& \left.+(1+\mu)^{2}\left[\left(a^{+}\right)^{3}-2\left(a^{-}\right)^{2}\left(a^{+}-a^{-}\right)\right] \frac{1}{4 \pi}\left\langle\frac{1}{|\vec{q}|}\right\rangle_{\mathrm{wf}}\right)+a_{\mathrm{boost}}+O\left(Q^{5}\right)
\end{aligned}
$$

where we have subsumed higher order effects into the $\pi N$ scattering lengths and $\langle\ldots\rangle_{w f}$ denotes a momentum space deuteron matrix element. Notice that since local two-pionfour-nucleon operators are not enhanced in the modified power counting they appear at the same order as in baryon CHPT, namely fifth order, and do not affect the fourth-order result given in Eq. (7). The $\pi^{-} d$ atomic level shift has been measured to high precision at PSI [24], yielding the complex-valued scattering length $a_{\pi d}=[-0.0261( \pm 0.0005)+$ $i 0.0063( \pm 0.0007)] M_{\pi}^{-1}$. One thus can analyze Eq. (7) using the real part of this number in the $\left(a^{+}-a^{-}\right)$-plane, as shown by the red bands in Fig. 6 . This theoretical uncertainty is due to the NLO EFT wave functions employed. If one uses the most recent and systematic determination of the pionic hydrogen level shift [18] (the green band in Fig. 6) together with the empirical constraint from the hydrogen width shown by the grey band in Fig. 6 
(which will be affected by further experimental [25] as well as theoretical corrections), one can determine the $\mathrm{S}-$ wave $\pi N$ scattering lengths

$a^{-}=[0.0936( \pm 0.0011)] M_{\pi}^{-1} ; \quad a^{+}=[-0.0029( \pm 0.0009)] M_{\pi}^{-1}$.

If one instead were to use the blue band for the hydrogen shift (based on incomplete potential model calculations), these numbers would read $a^{-}=[0.0917( \pm 0.0013)] M_{\pi}^{-1}$ and $a^{+}=[-0.0034( \pm 0.0008)] M_{\pi}^{-1}$. The theoretical error bars from both determinations should be considered somewhat optimistic given the neglect of isospin violation in [17]. For a comparison to other (more model-dependent) determinations and a discussion of the imaginary part of the $\pi d$ scattering length the reader is referred to [17]. For a first attempt towards a systematic calculation of pion- ${ }^{3}$ He scattering see [26].

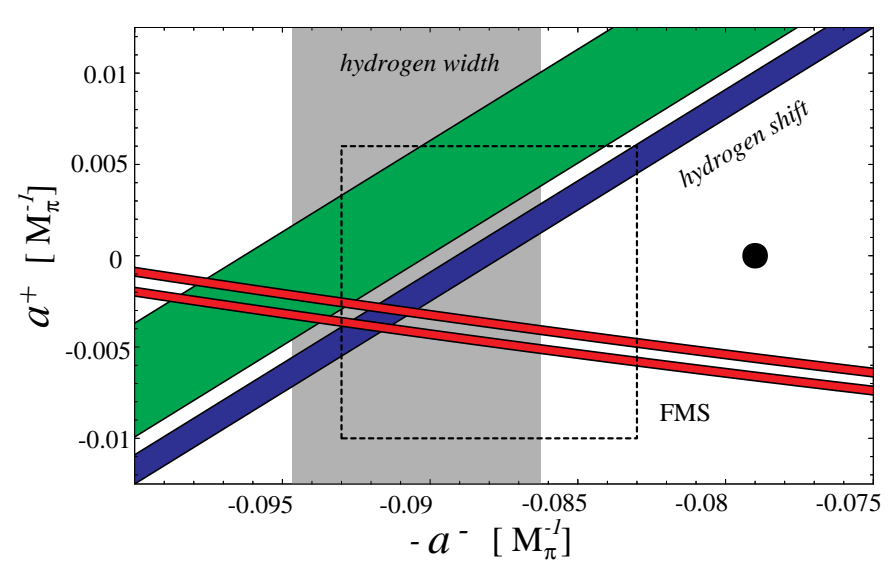

Figure 6. Determination of the $\mathrm{S}$ wave $\pi N$ scattering lengths from pionic hydrogen and deuterium as explained in the text. The heavy dot refers to the current algebra prediction and the black box to the CHPT determination from scattering data $[16,15]$.

\section{SUMMARY AND OUTLOOK}

I have outlined a program that allows to calculate nucleon and nuclear properties from one chiral effective field theory, based on a systematic power counting, embodying the symmetries of QCD and allowing for the estimation of theoretical uncertainties. As examples, I have discussed electron scattering off nucleons and deuterium and also pionnucleon and pion-deuteron scattering lengths, both cases displaying an intricate interplay between chiral nucleon and chiral nuclear dynamics. Other examples, which have gained some popularity over the last decade, are pion photo/electroproduction (see e.g. the discussion in [27] as well as [28] and [29]) and Compton scattering off nucleons [30] and light nuclei [31], to name just two. It remains to be seen how far this program can be carried out in terms of mass number $A$ (the extension to systems with $A=3,4$ is under way), photon virtuality $Q^{2}$ or pion energy $\omega$. One also might ask at what point other active degrees of freedom (the delta, vector mesons, ...) have to be taken into account explicitely? Many pioneering calculations have been done but much more work is required to really develop this scheme into a true working tool for precision nuclear, hadronic and few-body physics.

\section{Acknowledgements}

I thank the organizers for the invitation and their superb work and my collaborators, in particular Silas Beane, Véronique Bernard, Evgeny Epelbaum and Bastian Kubis, for 
sharing their insight into the various topics discussed here.

\section{REFERENCES}

1. At the frontier of particle physics - handbook of QCD, M Shifman (ed.), Vols. 1-4, World Scientific, Singapore, 2001-2002.

2. S.R. Beane, these proceedings.

3. E. Epelbaum, these proceedings.

4. N. Fettes, U.-G. Meißner, M. Mojžiš and S. Steininger, Annals Phys. 283 (2000) 273 [Erratum-ibid. 288 (2001) 249].

5. G. Ecker, J. Gasser, A. Pich and E. de Rafael, Nucl. Phys. B 321 (1989) 311.

6. J. F. Donoghue, C. Ramirez and G. Valencia, Phys. Rev. D 39 (1989) 1947.

7. V. Bernard, N. Kaiser and U.-G. Meißner, Nucl. Phys. A 615 (1997) 483.

8. E. Epelbaum, U.-G. Meißner, W. Glöckle and C. Elster, Phys. Rev. C 65 (2002) 044001.

9. V. Bernard, T. R. Hemmert and U.-G. Meißner, arXiv:hep-ph/0307115.

10. B. Kubis and U.-G. Meißner, Nucl. Phys. A 679 (2001) 698.

11. D. B. Kaplan, M. J. Savage and M. B. Wise, Phys. Rev. C 59 (1999) 617.

12. M. Walzl and U.-G. Meißner, Phys. Lett. B 513 (2001) 37.

13. D. R. Phillips, arXiv:nucl-th/0304046.

14. V. Bernard, N. Kaiser and U.-G. Meißner, Phys. Rev. C 52 (1995) 2185.

15. N. Fettes and U.-G. Meißner, Nucl. Phys. A 676 (2000) 311.

16. N. Fettes, U.-G. Meißner and S. Steininger, Nucl. Phys. A 640 (1998) 199.

17. S. R. Beane, V. Bernard, E. Epelbaum, U.-G. Meißner and D. R. Phillips, Nucl. Phys. A 720 (2003) 399.

18. J. Gasser, M. A. Ivanov, E. Lipartia, M. Mojzis and A. Rusetsky, Eur. Phys. J. C 26 (2002) 13.

19. N. Fettes and U.-G. Meißner, Nucl. Phys. A 693 (2001) 693.

20. S. Weinberg, Phys. Lett. B 295 (1992) 114.

21. S. R. Beane, V. Bernard, T. S. Lee and U.-G. Meißner, Phys. Rev. C 57 (1998) 424.

22. M. R. Robilotta and C. Wilkin, J. Phys. G 4 (1978) L115.

23. S. R. Beane and M. J. Savage, Nucl. Phys. A 717 (2003) 104.

24. P. Hauser et al., Phys. Rev. C 58 (1998) 1869.

25. D. Gotta [Pionic Hydrogen collaboration], arXiv:hep-ex/0305012.

26. V. Baru, J. Haidenbauer, C. Hanhart and J. A. Niskanen, Eur. Phys. J. A 16 (2003) 437.

27. U.-G. Meißner, V. Bernard, E. Epelbaum and W. Glöckle, arXiv:nucl-th/0301079.

28. V. Bernard, N. Kaiser and U.-G. Meißner, Eur. Phys. J. A 11 (2001) 209.

29. S. R. Beane, V. Bernard, T. S. Lee, U.-G. Meißner and U. van Kolck, Nucl. Phys. A 618 (1997) 381.

30. V. Bernard, N. Kaiser, J. Kambor and U.-G. Meißner, Nucl. Phys. B 388 (1992) 315.

31. S. R. Beane, M. Malheiro, J. A. McGovern, D. R. Phillips and U. van Kolck, arXiv:nucl-th/0209002. 